ARTICLE

DOI: $10.1038 / \mathrm{s} 41467-017-01760-5$

\title{
Structure of human immunoproteasome with a reversible and noncompetitive inhibitor that selectively inhibits activated lymphocytes
}

Ruda de Luna Almeida Santos (i) ${ }^{1}$, Lin Bai ${ }^{1}$, Pradeep K. Singh², Naoka Murakami ${ }^{3}$, Hao Fan ${ }^{4}$, Wenhu Zhan ${ }^{4}$, Yingrong Zhu ${ }^{4}$, Xiuju Jiang ${ }^{4}$, Kaiming Zhang ${ }^{5}$, Jean Pierre Assker ${ }^{3}$, Carl F. Nathan ${ }^{4}$, Huilin $\mathrm{Li}^{1}$, Jamil Azzi ${ }^{3} \&$ Gang $\mathrm{Lin}^{4}$

Proteasome inhibitors benefit patients with multiple myeloma and B cell-dependent autoimmune disorders but exert toxicity from inhibition of proteasomes in other cells. Toxicity should be minimized by reversible inhibition of the immunoproteasome $\beta 5 i$ subunit while sparing the constitutive $\beta 5 c$ subunit. Here we report $\beta 5$ i-selective inhibition by asparagineethylenediamine (AsnEDA)-based compounds and present the high-resolution cryo-EM structural analysis of the human immunoproteasome. Despite inhibiting noncompetitively, an AsnEDA inhibitor binds the active site. Hydrophobic interactions are accompanied by hydrogen bonding with $\beta 5 i$ and $\beta 6$ subunits. The inhibitors are far more cytotoxic for myeloma and lymphoma cell lines than for hepatocarcinoma or non-activated lymphocytes. They block human B-cell proliferation and promote apoptotic cell death selectively in antibodysecreting B cells, and to a lesser extent in activated human T cells. Reversible, $\beta 5 \mathrm{i}$-selective inhibitors may be useful for treatment of diseases involving activated or neoplastic B cells or activated T cells.

\footnotetext{
${ }^{1}$ Cryo-EM Structural Biology Laboratory, Van Andel Research Institute, Grand Rapids, MI 49503, USA. ${ }^{2}$ Department of Biochemistry and Milstein Chemistry Core Facility, Weill Cornell Medicine, New York, NY 10065, USA. ${ }^{3}$ Transplantation Research Center, Renal Division, Brigham and Women's Hospital, Harvard Medical School, Boston, MA 02115, USA. ${ }^{4}$ Department of Microbiology \& Immunology, Weill Cornell Medicine, New York, NY 10065, USA. ${ }^{5}$ National Center for Macromolecular Imaging and Marrs McLean Department of Biochemistry and Molecular Biology, Baylor College of Medicine, Houston, TX 77030, USA. Ruda de Luna Almeida Santos, Lin Bai, Pradeep K. Singh and Naoka Murakami contributed equally to this work. Correspondence and requests for materials should be addressed to H.L. (email: Huilin.Li@vai.org) or to J.A. (email: jazzi@rics.bwh.harvard.edu) or to G.L. (email: gal2005@med.cornell.edu)
} 
D egradation of most cytosolic proteins is a highly regulated, ATP-dependent cellular activity executed by the ubiquitin-proteasome system (UPS) ${ }^{1}$. The UPS plays essential roles in diverse cellular activities, including cell cycle control, signal transduction, protein homeostasis and immune surveillance. The degradation machinery of the UPS, the $26 \mathrm{~s}$ proteasome, is composed of a hydrolytic barrel-like $20 \mathrm{~s}$ core and regulators, such as $19 \mathrm{~s}$ or $11 \mathrm{~s}$, on either or both ends of the $20 \mathrm{~s}$. The $20 \mathrm{~s}$ core that is constitutively expressed in most cells (c-20S) is a stack of 4 rings of $14 \alpha$ and $14 \beta$ subunits organized in a $\alpha_{1-7} \beta_{1-7} \beta_{1-7} \alpha_{1-7}$ fashion, where 2 copies of each caspase-like $\beta 1$, trypsin-like $\beta 2$ and chymotrypsin-like $\beta 5$ active subunit are located in the inner $\beta$ rings $^{2}$. The chymotrypsin-like $\beta 5$ active subunit of the $20 \mathrm{~s}$ has been clinically validated as a target for the treatment of multiple myeloma and certain lymphomas. The Food and Drug Administration-approved drugs bortezomib and carfilzomib represent two classes of covalent proteasome inhibitors: reversible peptide boronates and irreversible peptide epoxyketones, respectively ${ }^{3}$. Several other classes of proteasome inhibitors have been identified and optimized, such as $\beta$-lactones and peptide sulfonyl fluorides ${ }^{4}$. However, their reactive warheads pose a challenge for developing a drug candidate. Several noncompetitive proteasome inhibitors have recently been reported $^{5-7}$.

We have been developing isoform-selective, non-covalent inhibitors for various proteasomes, such as the Mycobacterium tuberculosis proteasome ${ }^{8-12}$ and the human immunoproteasome $(\mathrm{i}-20 \mathrm{~s})^{13-15}$. I-20S is expressed in cells of the immune system and other cells exposed to cytokines that are elevated during immune responses, where the active subunits $\beta 1 c, \beta 2 c$, and $\beta 5 c$ in $c-20 S$ are replaced by $\beta 1 \mathrm{i}, \beta 2 \mathrm{i}$ and $\beta 5 \mathrm{i}$, respectively ${ }^{16-18}$. The $\mathrm{i}-20 \mathrm{~S}$ serves diverse functions in the immune system, including the provision of oligopeptides for antigen presentation, $\mathrm{T}$ cell differentiation and proliferation ${ }^{19,20}$. Antibody-secreting plasma cells are highly sensitive to proteasome inhibition. Bortezomib, which inhibits both c-20s and i-20S, has been used in renal transplant recipients to treat antibody-mediated graft rejection ${ }^{21}$. Bortezomib was also reported to be efficacious in patients with refractory systemic lupus erythematosus ${ }^{22}$. However, bortezomib's substantial mechanism-based toxicity requires use of much reduced doses in the treatment of non-malignant conditions.

To regulate immune responses through proteasome inhibition with less mechanism-based toxicity to immune cells and little or none to other cells, it would be useful to inhibit i-20S selectively, sparing c-20S. Consistent with this notion, and unlike disruption of genes encoding c-20S subunits, disruption of genes encoding $\beta 1 \mathrm{i}, \beta 2 \mathrm{i}$ and $\beta 5 \mathrm{i}$ results in mice that are healthy, fertile and immunocompetent ${ }^{23}$. Indeed, relatively selective inhibition of $\beta 5 \mathrm{i}$ over $\beta 5 \mathrm{c}$ with the compound ONX-0914 has been efficacious in several mouse models of autoimmune disease ${ }^{24}$. However, ONX0914 belongs to the peptide epoxyketone class of inhibitors whose irreversible mechanism involves recruiting the hydroxyl and amino groups of the active site $\mathrm{Thr}^{1 \mathrm{~N}}$ into formation of a 1,4oxazepane adduct with the epoxyketone warhead. Long-term use of an irreversible inhibitor presents a risk of toxicity from the gradual, cumulative inhibition of c-20S and potentially of other targets. Therefore, it would be desirable to develop inhibitors that are highly selective for i-20S as well as reversible ${ }^{25}$. Several recent

a<smiles>CC(C)(C)OC(=O)CN(C(=O)NC(=O)CCc1ccccc1)C(=O)C(NC(=O)Cc1cccc2ccccc12)c1ccc(F)cc1</smiles>

b
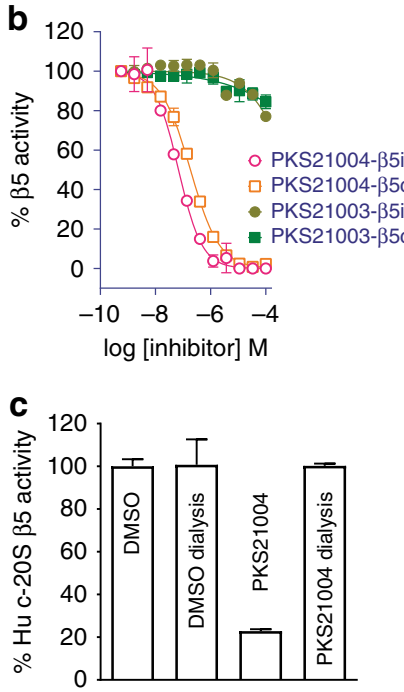<smiles>CC(C)(C)NC(=O)CC(NC(=O)CCc1ccccc1)C(=O)NCCNC(=O)c1cccc(-c2ccccc2)c1</smiles>
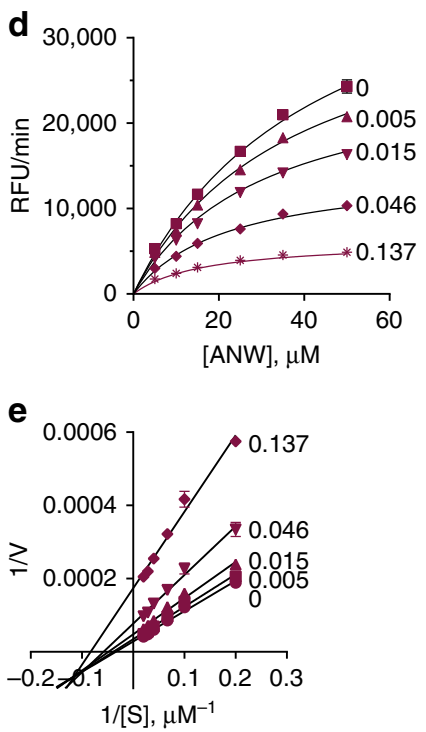

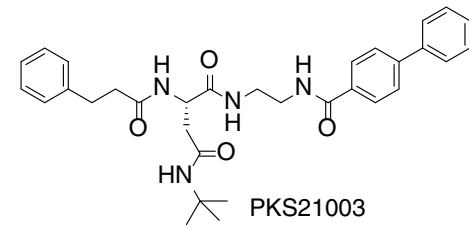

f

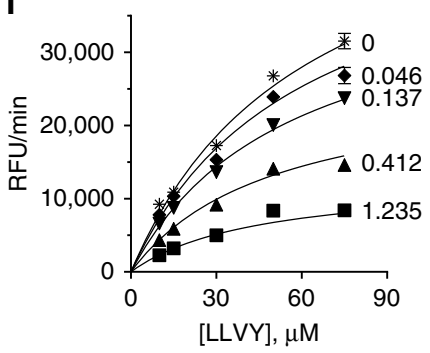

g

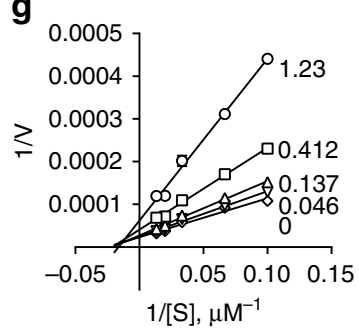

Fig. 1 AsnEDAs noncompetitively inhibit $\beta 5 i$ and $\beta 5 c$. a Dipeptide DPLG3 and AsnEDA PKS2100 4 and PKS21003. b PKS21004 potently and dosedependently inhibits $\beta 5 \mathrm{i}$ and $\beta 5 \mathrm{c}$, whilst PKS21003 is inactive. $\mathbf{c}$ PKS21004 reversibly inhibits $\beta 5 \mathrm{c}$. $\mathbf{d}-\mathbf{g}$ kinetics studies of modality of inhibition for PKS21004 against $\beta 5 i(\mathbf{d}, \mathbf{e})$ and $\beta 5 c(\mathbf{f}, \mathbf{g})$. d Steady state velocities in the presence of PKS21004 at the concentrations indicated next to each curve. Data as in $\mathbf{d}$ are shown in double reciprocal plots in $\mathbf{e}$, and data from $\mathbf{f}$ in $\mathbf{g}$. Values of Ki and $\alpha$ for PKS21004 were determined to be $0.077 \mu \mathrm{M}$ and $0.28 \mu \mathrm{M}$ for $\beta 5 \mathrm{i}$, and $0.55 \mu \mathrm{M}$ and 0.65 for $\beta 5 \mathrm{c}$, respectively, by fitting the data to an equation for noncompetitive inhibitors. Data are means \pm SEM of three independent experiments 

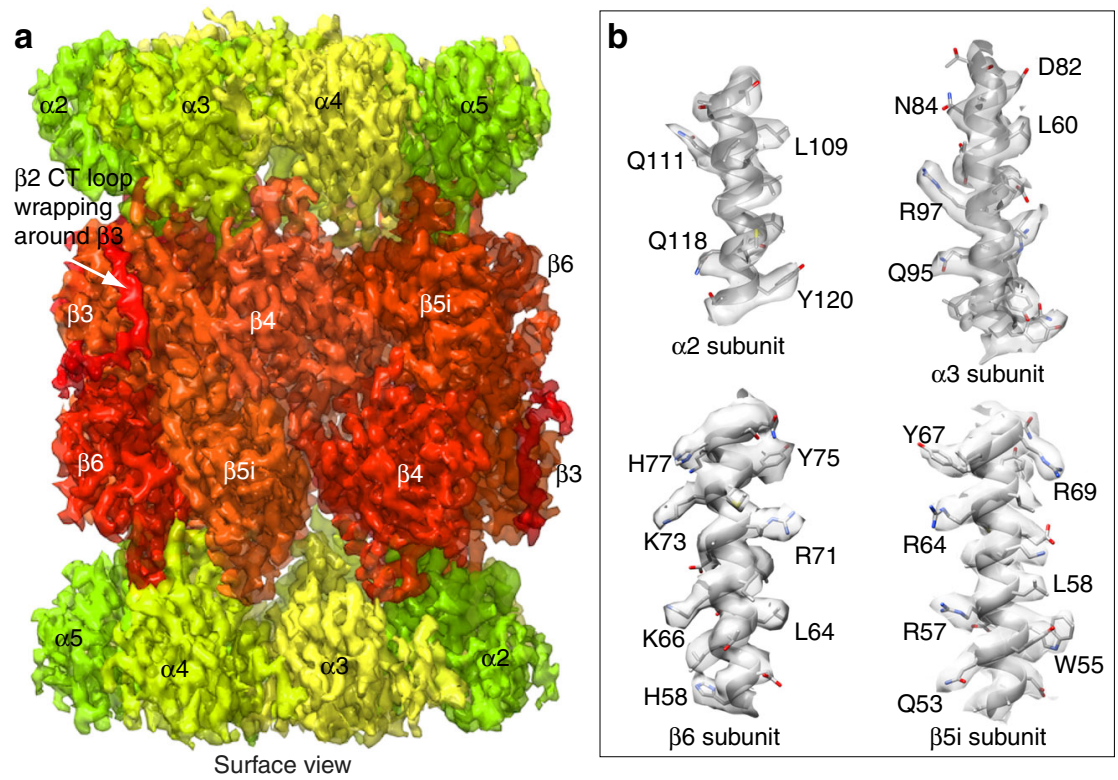

c
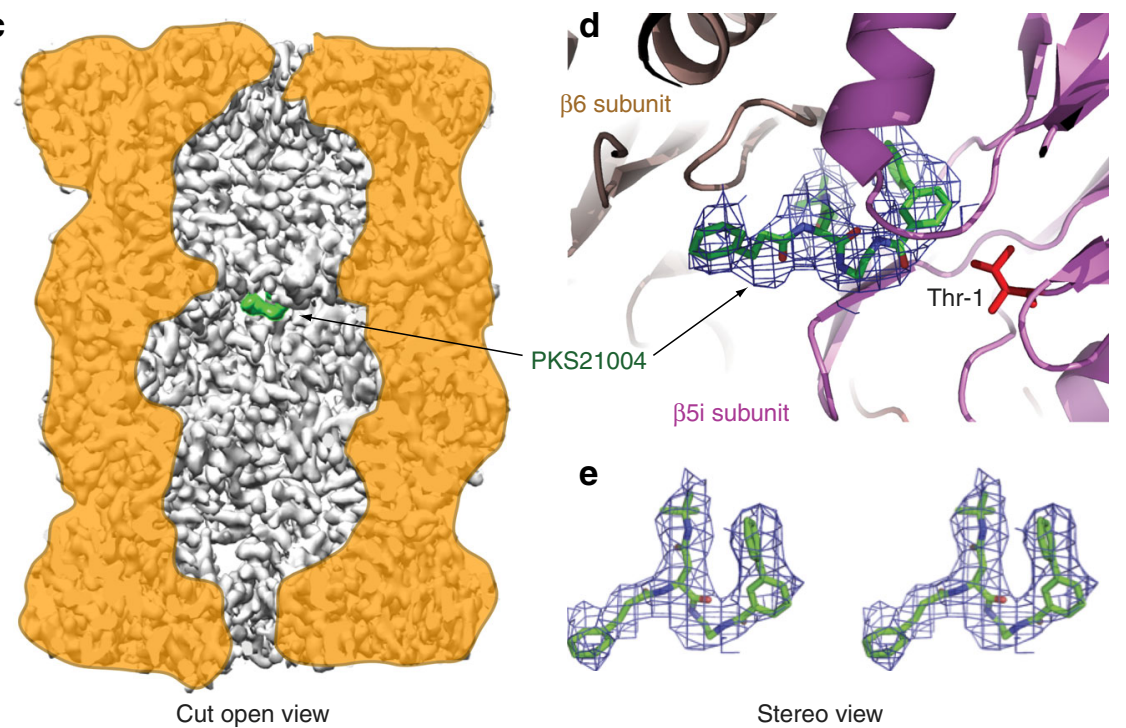

Stereo view

Fig. 2 Structure of the human i-20S bound to PKS21004, determined by high-resolution cryo-EM and single-particle analysis. a Surface view of the cryoEM map with each subunit colored differently. $\mathbf{b}$ Electron densities of four selected $\alpha$-helices demonstrating the resolution of many side chains of the i-20S complex. c A vertical central section of the 3.8- $\AA$ cryo-EM density map rendered as surface view. The inhibitor density is highlighted in green. $\mathbf{d}$ The electron density of the PKS21004 in the $\beta 5 i$ active site shown as blue meshes with modeled atomic structure shown as green sticks. The structure of the $\beta 5 \mathrm{i}$ is shown as cartoon in magenta and $\beta 6$ in salmon, respectively. The proteolytic residue Thr 1 of $\beta 5 \mathrm{i}$ is in red sticks. e A stereo view of the binding pose of PKS21004

studies have reported the development of $\beta 5 \mathrm{i}$-selective inhibitors $^{26,27}$. Because of the abundance of proteasomes' substrates, an additional benefit might accrue from a noncompetitive mode of action, so that progressive accumulation of substrate does not lessen the degree of inhibition. Herein we report the serendipitous discovery of a class of non-covalent compounds that noncompetitively and selectively inhibit $\beta 5 \mathrm{i}$ over $\beta 5$ c.

\section{Results}

Scaffold morphing from dipeptides to AsnEDAs. We recently reported a class of irreversible inhibitors that selectively inhibit the Mycobacterium tuberculosis proteasome over human c-20S ${ }^{11}$. We later found that this class of inhibitors also selectively inhibits $\mathrm{i}-20 \mathrm{~S}$ over $\mathrm{c}-20 \mathrm{~S}^{13}$, reflecting that the mycobacterial and human $\mathrm{i}-20 \mathrm{~S}$ proteasomes share similar folding around the $\beta 5 \mathrm{i}$ active subunit. We later identified non-covalent N,C-capped dipeptides with bulky hydrophobic aromatic rings at P1 and $\mathrm{N}, \mathrm{N}$ - dual substituted asparagine side chains that are highly potent and selective for Mtb20S over both human c-20S and i-20S ${ }^{12,28}$. We then developed a class of N,C-capped dipeptidomimetics by incorporating a $\beta$-amino acid into the $\mathrm{N}, \mathrm{C}$-capped dipeptides, which resulted in markedly potent and selective inhibitors for $\beta 5 \mathrm{i}$ over $\beta 5 c^{15}$. We also developed $\beta 5 i$-selective N,C-capped dipeptides that suppressed T-cell activation and proliferation in vitro and helped prevent rejection of cardiac allografts in mice ${ }^{14}$.

To move away from peptides, we systematically replaced the amide bonds in select N,C-capped dipeptides with bioisosteres. Reversing the amide bond at the C-cap and replacing the amino acid with an aromatic carboxylic acid resulted in a chemotype: Asn-ethylenediamine (AsnEDA) (Fig. 1a). The first compound of 
Table 1 Cryo-EM data collection and refinement statistics

\begin{tabular}{lc} 
Human i-20S-PKS21004 (EMD-7010, PDB 6AVO) & \\
\hline Data collection & \\
EM equipment & JEOL JEM-3200FS \\
Voltage $(\mathrm{kV})$ & 300 \\
Detector & Gatan K2 Summit \\
Pixel Size $(\AA)$ & 1.2 \\
Electron Dose $\left(\mathrm{e}^{-} \AA^{-2}\right)$ & 60 \\
Underfocus range ( $\mu \mathrm{m})$ & $1.0-3.0$ \\
Reconstruction & \\
Software & RELION 2.0 \\
Number of particles used & 75,017 \\
Resolution $(\AA)$ & 3.8 \\
Map-sharpening B factor $\left(\AA^{2}\right)$ & 124.3 \\
Model composition & \\
Peptide chains & 28 \\
Protein residues & 6115 \\
Ligands & 2 \\
R.m.s deviation & \\
Bond length & 0.009 \\
Bond angle & 1.146 \\
Ramachandran plot & \\
Preferred (\%) & 90.70 \\
Allowed (\%) & 9.22 \\
Outlier (\%) & 0.08 \\
Validation & \\
MolProbity score & 2.52 \\
Good rotamer (\%) & 98.02 \\
Clashscore, all atoms & 32.47 \\
\hline
\end{tabular}

this class, PKS3080, yielded modest IC50s of 0.37 and $1.22 \mu \mathrm{M}$ against human $\beta 5 \mathrm{i}$ and $\beta 5 \mathrm{c}$, respectively. Replacement of the 1naphthoyl with 2-naphthoyl (producing PKS21025) improved potency against $\beta 5 \mathrm{i}$ by 3 -fold and increased selectivity to 26 -fold.

Replacement of the $N$-cap 1-naphthoyl with [1,1'-biphenyl]-4carboxamide (PKS21003) and [1,1'-biphenyl]-3-carboxamide (PKS21004) drastically impacted activity. PKS21003 had no detectable activity against either $\beta 5 \mathrm{i}$ or $\beta 5 \mathrm{c}$ (IC50 $>100 \mu \mathrm{M}$ for both), whereas PKS21004 was a potent inhibitor of both $\beta 5 \mathrm{i}$ and $\beta 5 c$, with IC50s of 0.058 and $0.326 \mu \mathrm{M}$, respectively (Fig. 1b). Inhibition of the $\beta 5$ subunits was specific, as no inhibition was observed of $\beta 1$ or $\beta 2$ activities in either i-20S or c-20S (Supplementary Table 1).

Modality of inhibition. As expected from their non-covalent chemistry, dialysis of a pre-incubated mixture of c-20S and PKS21004 fully restored $\beta 5 \mathrm{c}$ activity (Fig. 1c), confirming the reversibility of this class of inhibitors. Kinetic analysis indicated that PKS21004 inhibits $\beta 5 i$ and $\beta 5 c$ noncompetitively with substrate. With increasing concentrations of PKS21004, both $V_{\max }$ and $K_{\mathrm{M}}$ decreased (Fig. $1 \mathrm{~d}-\mathrm{g}$ and Supplementary Fig. 1). This indicates that inhibition of $\beta 5 \mathrm{i}$ and $\beta 5 \mathrm{c}$ by PKS21004 is of a mixed noncompetitive type with $\alpha_{\mathrm{c}-20 \mathrm{~S}} \approx 0.65$ and $\alpha_{\mathrm{i}-20 \mathrm{~S}} \approx 0.28^{29}$, and that PKS21004 binds more tightly to both $\beta 5 \mathrm{c}$ and $\beta 5 \mathrm{i}$ with substrate bound than without substrate (Supplementary Fig. 1). Decreasing $V_{\max } / K_{\mathrm{M}}$ with increasing PKS21004 concentration also suggests that PKS21004 is not an uncompetitive inhibitor of either 20S (Supplementary Fig. 1) ${ }^{29}$. This class thus joins a growing list of noncompetitive proteasome inhibitors ${ }^{5,30}$.

Cryo-EM structure of human i-20S with PKS21004. To identify the binding site of the compound, we used high-resolution cryoelectron microscopy and single-particle analysis to determine the structure of the human immunoproteasome in complex with PKS21004 (See Experimental methods). The final cryo-EM 3D map had a resolution of $3.8 \AA$ in which the secondary structures such as $\beta$-strands and $\alpha$-helices were very well resolved (Fig. 2a, Supplementary Figs. 2-6). Many large side chains were also resolved (Fig. 2b). The large side chain densities, in conjunction with the previously published crystal structures of the human constitutive proteasome (PDB code 5LF1) $)^{25}$ and the mouse immunoproteasome (PDB code $3 \mathrm{UNH})^{31}$, enabled us to build an atomic model at this resolution (see Table 1 for model statistics). In the electron density map and the atomic model, the very long carboxyl terminal extension of the human $\beta 2 \mathrm{i}$ embraces nearly half of the $\beta 3$ subunit (Fig. 2a); this feature is highly conserved from yeast to humans (Supplementary Fig. 7).

We were able to unambiguously assign the density in the substrate-binding cleft near the active site of $\beta 5$ i to PKS21004 (Fig. 2c-e). No additional densities were observed in the active site of any other $\beta$-subunits or in any of the $\alpha$ - subunits. This result is consistent with the conclusion that PKS21004 specifically inhibits the activity of the $\beta 5$ subunits. PKS21004 binds to human $\mathrm{i}-20 \mathrm{~S}$ between $\beta 5 \mathrm{i}$ and $\beta 6$ at a position similar to where the covalent inhibitor dihydroeponemycin binds in the $\beta 1 c, \beta 2 c$, and $\beta 5 \mathrm{c}$ of the human c-20S (PDB code 5LF1) ${ }^{25}$.

PKS21004 is largely hydrophobic and interacted with $\beta 5 \mathrm{i}$ and $\beta 6$ primarily via hydrophobic contacts (Fig. 3a-b, Supplementary Fig. 8A, B). Specifically, the R1 group extended deeply into the S1 and S1 sub pocket (S1SP), forming extensive hydrophobic contacts with Ala20, Val31, Ser46, Gly47, Ala49, and Gln53 of $\beta 5 \mathrm{i}$, and Tyr130 of $\beta 6$; the R2 group, being outside of the active site, interacted with Ser21, Ala22, and Cys48 of $\beta 5 \mathrm{i}$, and Val127 of $\beta 6$; the R3 group was next to the $\beta 6$ subunit, forming interactions with Ala28 of $\beta 5$ i, and Ser123, Phe124, Ser129, and Gln131 of $\beta 6$. The inhibitor also formed two hydrogen bonds, one between the R3 group and Ser27 of $\beta 5 \mathrm{i}$, and the other between R3 and Asp125 of $\beta 6$. The overall structures of the catalytic $\beta 1 \mathrm{i}, \beta 2 \mathrm{i}$, and $\beta 5 \mathrm{i}$ are very similar to their constitutive counterparts, except for several minor differences in the loop regions (Supplementary Fig. 9A). However, among the human $\beta 5 \mathrm{i}$ residues interacting with PKS21004, many are different from their corresponding residues in the human $\beta 5 \mathrm{c}$ (Supplementary Figs. 8, 9B). Notably, Ala27 and Ser53 in $\beta 5 c$ are replaced by Ser27 and Gln 53 in $\beta 5 i$, thus disrupting two important interactions with PKS21004 in the $\beta 5 \mathrm{i}$ (Fig. 3c, Supplementary Fig. 8A, C). The side chain of Met 45 is swung outward as compared to the apo human $\beta 5 c$ or $\beta 5 c$ in complex with CFZ to accommodate the bulky R1 group in $\beta 5 i$. (Supplementary Fig. 10). These differences may explain why PKS21004 selectively inhibits i-20S over c-20S.

The seeming discrepancy between kinetic and structural studies indicates that PKS21004 belongs to a class of active sitedirected noncompetitive inhibitors, along with certain inhibitors of other enzymes, especially kinases ${ }^{32}$. However, to our knowledge, this is the example of an active site-directed noncompetitive inhibitor of proteasomes.

Optimization of AsnEDAs. The foregoing features of these AsnEDA compounds encouraged a further round of SAR studies (Table 2) in which we varied the carboxylic acid at the ethylenediamine, the $N$-cap at the Asn, and the side chain of Asn, of PKS21004. All compounds listed in Table 1 were synthesized as illustrated in Scheme 1-9 in Supplementary Methods. The structures of all final compounds were confirmed by nuclear magnetic resonance (NMR) and high-resolution mass spectroscopy (HRMS). $\mathrm{IC}_{50} \mathrm{~s}$ of all compounds against $\beta 5 \mathrm{i}$ and $\beta 5 \mathrm{c}$ (Table 2) and against $\beta 1 \mathrm{i}, \beta 2 \mathrm{i}, \beta 1 \mathrm{c}$, and $\beta 2 \mathrm{c}$ (Supplementary Table 1) were determined following reported methods ${ }^{8}$. All compounds were specific for the $\beta 5$ subunit; no inhibition of $\beta 1 \mathrm{i}$, $\beta 1 c, \quad \beta 2 \mathrm{i}, \quad$ or $\beta 2 \mathrm{c}$ was observed. Comparison of the 

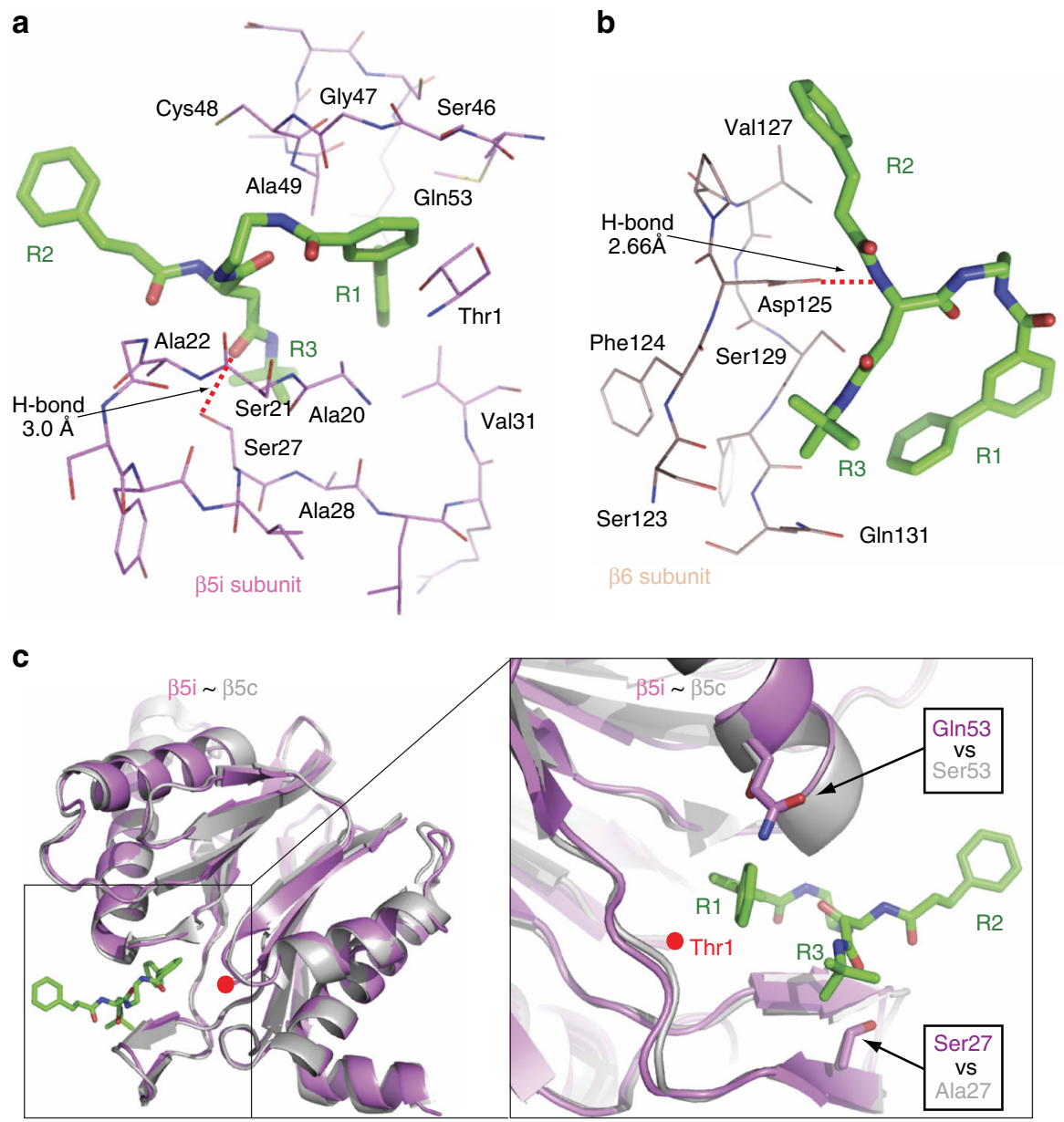

Fig. 3 The interaction of PKS21004 with the human i-20S. a Detailed hydrophobic interactions between the R1 and R3 groups of PKS21004 with the substrate pocket of the human $\beta 5 \mathrm{i}$ subunit. A hydrogen bond between Ser27 and the oxygen atom of the PKS21004 R3 group (3.0 $\AA$ ) is shown as a dashed red line. $\mathbf{b}$ Interactions of the R2 and R3 groups of PKS21004 with the neighboring $\beta 6$ subunit. The strong $\mathrm{H}$-bond between Asp125 and the nitrogen atom of the R2 of PKS21004 ( $2.66 \AA$ ) is shown by a dashed red line. c Representation of the PKS21004 (green sticks) in the $\beta 5 i$ active site (purple cartoon). The structure of $\beta 5$ (gray cartoon, PDB 5LF1) is superimposed for comparison. The right panel is an enlarged and rotated view of the area in the square box of the left panel. Red dots denote the active residue Thr1 of $\beta 5$ and $\beta 5$ i. The two residues in human $\beta 5 i$ that make contact with the inhibitor and are different from the human $\beta 5 \mathrm{c}$ are shown in stick and marked by two black arrows

ethylenediamine with a methyl-ethylenediamine (PKS21018) and a 1,3-propyldiamine (PKS21019) indicated that the ethylenediamine gave the greatest potency for $\beta 5 \mathrm{i}$ and selectivity over $\beta 5 \mathrm{c}$. We then modified the $\left[1,1^{\prime}\right.$-biphenyl]-3-carboxamide of PKS21004 with the following substituents: 4'-F (PKS21026), 4'cyano (PKS21028), 4-F (PKS21196), 4,3'-diF (PKS21195) and 4,2'-diF (PKS21187). The $4^{\prime}$-substitutions decreased potency, while 4- and 4,3'- substitutions did not. Best, 4,2'-diF(PKS21187) improved potency against $\beta 5 \mathrm{i}$ to IC50 $0.015 \mu \mathrm{M}$ and improved selectivity to $\sim 20$-fold over inhibition of $\beta 5 \mathrm{c}$. Consistent with these results, the binding pocket in the cryo-EM structure is large enough to accommodate the new fluoride in 4- and 4,3'positions. Notably, two crucial residues around these sites, Ser 46 and Gln53 in $\beta 5$ i, are changed to Ala 46 and Ser53 in $\beta 5 c$, which may account for the improved potency of PKS21187 against $\beta 5 \mathrm{i}$ over $\beta 5 \mathrm{c}$ (Supplementary Fig. $8 \mathrm{~A}-\mathrm{D}$ ).

Next, we investigated a $N$-Boc-Asp- ${ }^{t} \mathrm{Bu}$ substitution on PKS21277, an intermediate in the synthesis of PKS21187. Compared with PKS21187, PKS21277 has a smaller R2 group, which may abolish the interactions with Val127 in $\beta 6$ and Cys48 in $\beta 5 \mathrm{i}$ that is changed to Gly 48 in $\beta 5 \mathrm{c}$ (Supplementary Fig. $8 \mathrm{~A}-\mathrm{C}$ ). Consistently, PKS21277 was modestly potent against $\beta 5 \mathrm{i}$, with 36 fold selectivity over $\beta 5 c$ (Table 2, Supplementary Fig. 8D).
Replacing the Asp- ${ }^{t} \mathrm{Bu}$ with Gly (PKS21276), Ala (PKS21280), or Asn (PKS21281) abolished the inhibitory activities against both $\beta 5 \mathrm{i}$ and $\beta 5 c$, suggesting that $A s p-{ }^{t} \mathrm{Bu}$ is critical for optimal binding to $\beta 5$ (Supplementary Fig. 8). We then replaced phenylpropionate with tosyl on the $N$-cap of the Asn of PKS21187, yielding PKS21221. Based on the cryo-EM structure, the tosyl group may form tighter interactions with Ser27 and Cys48 in the human $\beta 5$ i (Supplementary Fig. 8). IC50s were determined to be $4 \mathrm{nM}$ against $\beta 5 \mathrm{i}$ and $110 \mathrm{nM}$ against $\beta 5 \mathrm{c}$, representing 30-fold selectivity. Again, replacing the Asp- ${ }^{t} \mathrm{Bu}$ of PKS21221 with Gly (PKS21289), Ala (PKS21290), or Asn (PKS21288) eliminated inhibitory activity against both $\beta 5 \mathrm{i}$ and $\beta 5 c$. We then replaced the tosyl of PKS21221 with acetyl which yielded PKS21294. PKS21294 showed much reduced potency in comparing to PKS21221 by 150 -fold against $\beta 5$ i, by 120 -fold against $\beta 5 \mathrm{c}$. Replacing the tosyl of PKS21221 with cyclopropanesulfonyl, yielded PKS21293 that showed 3.7-fold reduction in potency against $\beta 5 \mathrm{i}$ in comparing to PKS21221. However, its selectivity against $\beta 5 \mathrm{i}$ over $\beta 5 \mathrm{c}$ was improved from 27.5 -fold to 64-fold.

To corroborate that the noncompetitive modality of inhibition was shared among this class of compounds, we tested PKS21221 against $\beta 5 i$ and confirmed the noncompetitive mechanism; $\alpha$ was 
Table 2 SAR studies and IC50s of compounds against human $\beta 5 i$ and $\beta 5 c$

\begin{tabular}{|c|c|c|c|c|c|}
\hline \multirow{2}{*}{ ID } & \multicolumn{2}{|c|}{$R_{2} \cdot N^{R_{3}} N_{N} R_{0}^{R_{1}}$} & \multicolumn{3}{|c|}{ IC50 $(\mu \mathrm{M})$} \\
\hline & $\mathrm{R}_{2}$ & $\mathrm{R}_{3}$ & $\mathrm{R}_{1}$ & $\beta 5 i$ & $\beta 5 c$ \\
\hline PKS3080 & $a_{0}^{0}$ & - i ist & $\cdot 8$ & 0.37 & 1.22 \\
\hline PKS21025 & $a_{0}$ & - $i_{A} \downarrow$ & $\infty$ & 0.091 & 2.607 \\
\hline PKS21003 & $a_{0} \cdot$ & - $i_{A} t$ & $\cdot \square$ & $>100$ & $>100$ \\
\hline PKS21004 & $a_{0}$ & - $i_{H} \downarrow$ & & 0.058 & 0.326 \\
\hline PKS21018 & & a tyoly & & 2.35 & 13.93 \\
\hline PKS21019 & & 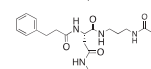 & & 0.28 & 3.05 \\
\hline PKS21026 & $a_{0}^{\circ}$ & - $i_{A} \leftarrow$ & & 0.11 & 1.16 \\
\hline PKS21028 & $a_{0}$ & - $i_{n} \leftarrow$ & & 2.07 & 7.25 \\
\hline PKS21196 & $a_{0}$ & - $i_{A} \leftarrow$ & & 0.044 & 0.68 \\
\hline PKS21195 & $a_{0}^{0}$ & - $i_{A} \neq$ & & 0.059 & 1.94 \\
\hline PKS21187 & $a_{0}$ & - int & & 0.016 & 0.36 \\
\hline PKS21277 & $x^{0} r_{0}^{0}$ & - $i_{\mu}+$ & & 0.11 & 3.80 \\
\hline PKS21276 & $x^{0}{ }^{0}$ & $\mathrm{H}$ & 5 & 24.25 & $>100$ \\
\hline PKS21280 & $x^{0} r_{0}^{0}$ & $\mathrm{CH}_{3}$ & & 33.23 & $>100$ \\
\hline PKS21281 & $x^{0} r_{0}^{0}$ & - $i_{\mathrm{NH}_{2}}$ & & $>100$ & $>100$ \\
\hline PKS21284 & H & - $i_{H} t$ & & 1.0 & 3.84 \\
\hline PKS21221 & तes: & - i i & 5 & 0.004 & 0.11 \\
\hline PKS21289 & $\mathbb{T}_{\gamma_{0}}$ & $\mathrm{H}$ & & 40.3 & 60.8 \\
\hline PKS21290 & Th. & $\mathrm{CH}_{3}$ & & $>100$ & $>100$ \\
\hline PKS21288 & 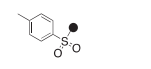 & - $i_{\mathrm{NH}_{2}}$ & Fis & 4.14 & $>100$ \\
\hline PKS21294 & $\mathrm{CH}_{3} \mathrm{CO}-$ & - i i $t$ & Fing & 0.62 & 13.26 \\
\hline PKS21293 & $\underset{\delta_{0}}{\Delta S_{0}}$ & - $i_{A} t$ & .126 & 0.015 & 0.96 \\
\hline
\end{tabular}

All IC50s are means of at least three independent experiments, except that IC50s greater than $10 \mu \mathrm{M}$ are means of two independent experiments

determined to be 0.26 (Supplementary Fig. 11), in agreement with that of PKS21004.

PKS21221 selectively induces cell death in MM cells. To determine if this class of inhibitors is cell penetrable, we treated the B-cell lymphoma line Karpas $1106 \mathrm{P}^{28}$ (expressing a high proportion of i-20S over c-20S) with PKS21221 before incubation with either (Ac-ANW) $)_{2}$-R110 (a specific substrate of $\beta 5 \mathrm{i}$ ) or sucLLVY-luciferin (a substrate of $\beta 5$ ) (Fig. 4a). IC50 values with both substrates were almost identical (172 and $118 \mathrm{nM}$ ) and indicated the cell-penetrating ability of these compounds. Similarly, PKS21221 inhibited $\beta 5 c$ activity in HepG2 hepatoma cells with an
IC50 of $1833 \mathrm{nM}$ (Table 3). No $\beta 5 \mathrm{i}$ activity was detected in HepG2 cells using (Ac-ANW) $)_{2}$-R110 as a substrate. PKS21221 itself does not inhibit luciferase activity at $100 \mu \mathrm{M}$ when tested over the range $0.002-100 \mu \mathrm{M}$.

Multiple myeloma cells are hypersensitive to inhibition of chymotryptic activity of their proteasomes and the panproteasome inhibitor bortezomib has clinical benefit in patients with multiple myeloma ${ }^{33,34}$. We incubated MM.1S, RPMI 8226, Karpas and HepG2 cells with PKS21221 at different concentrations, and determined cell viability after $72 \mathrm{~h}$ (Fig. 4b). PKS21221 showed potent cytotoxicity against the myeloma cell lines MM.1S and RPMI 8226, but was $\sim 10$-fold and $\sim 30$-fold less 

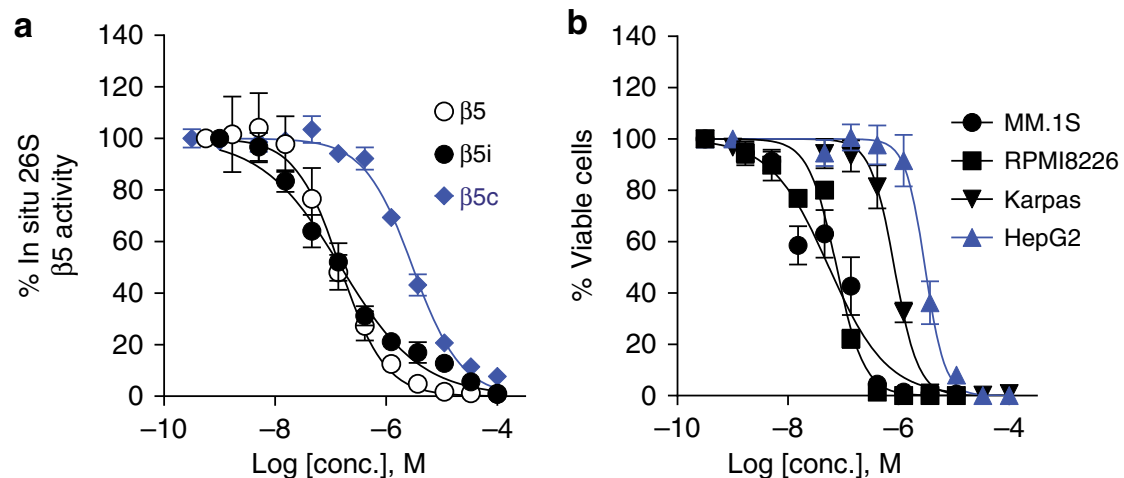

Fig. 4 Intracellular proteasome inhibition by PKS21221 and its cytotoxicity against transformed cell lines. a Karpas 1106P lymphoma cells (white and black symbols) or HepG2 cells (blue symbols) were treated with PKS21221 for $2 \mathrm{~h}$ at the concentrations indicated prior to incubation with substrate (AcANW) ${ }_{2}$ R110 for $\beta 5$ i (Karpas cells) or suc-LLVY-luciferin for $\beta 5$ (Karpas cells and HepG2 cells). IC50s were 0.154 and $0.149 \mu$ M for the two substrates in Karpas cells. b Cytotoxicity of PKS21221 against multiple myeloma cell lines MM.1S (solid circle) and RPMI 8226 (solid square), Karpas (solid triangle) and HepG2 (blue triangle). Cell viability was determined by CellTiter-glo. Values of IC50 of intracellular proteasome inhibition and EC50 of cytotoxicity are listed in the Table 3. Data are means \pm SEM of three to four independent experiments

cytotoxic against the Karpas lymphoma cell line and the HepG2 hepatocarcinoma cell line cells, respectively (Table 3).

PKS21221 is selectively cytotoxic to ASC's. The immunoproteasome plays important roles in $\mathrm{T}$ cell differentiation and proliferation and dendritic cell activation and maturation ${ }^{14,20,24}$. However, its role in B cell differentiation is not well understood. The pan-proteasome inhibitor bortezomib depleted plasma cells in mice with lupus-like disease ${ }^{35}$. To investigate whether an immunoproteasome-selective inhibitor impacts the survival of $\mathrm{B}$ cells, especially differentiated antibody-secreting cells (ASCs), we differentiated $\mathrm{B}$ cells in human peripheral blood mononuclear cells (PBMCs) into $\mathrm{CD} 19^{+} \mathrm{CD} 38^{+} \mathrm{CD} 27^{+}$ASCs in the presence of IL-2 and toll-like receptor (TLR)7/8 agonist R848 for 5 days $^{36,37}$. Differentiated ASCs were then exposed to PKS21221 at indicated concentrations and cell survival was determined with Annexin V and 7-aminoactinomycin D (7-AAD) at three-time points (12-h, 24-h, and 48-h after treatment). The percentage of viable ASC decreased with PKS21221 treatment in a dose- and time-dependent manner (Fig. 5a, Supplementary Fig. 12b, c), while the overall viability of PBMCs was not affected (Fig. 5b, Supplementary Fig. 12D). This was explained by enhanced apoptosis (Fig. 5c, d, left) and cell death (Fig. 5c, d, right) of the ASCs. Apoptosis of $\mathrm{CD} 9^{+}$non-ASCs was slightly enhanced but no increase was observed in apoptosis of $\mathrm{CD}^{-} 9^{-}$cells (Fig. 5e, left). Similarly, PKS21221 treatment did not affect viability of $\mathrm{CD}_{1}{ }^{-}$cells or $\mathrm{CD} 19^{+}$non-ASCs (Fig. 5e, right). These results suggest that PKS21221 specifically affected the viability of ASCs and to a lesser extent, non-antibody-secreting CD19 ${ }^{+} \mathrm{B}$ cells. In addition, the cytotoxicity of PKS21221 to different cell populations in PBMC was examined and compared to the cytotoxicity of bortezomib. PBMCs were incubated with PKS21221 or bortezomib, and the viability was assessed in $\mathrm{T}$ cells $\left(\mathrm{CD}^{+}\right)$, B cells $\left(\mathrm{CD} 19^{+}\right)$, monocytes $\left(\mathrm{CD} 14^{+}\right)$and dendritic cells $\left(\mathrm{CD}^{-} \mathrm{CD} 19^{-} \mathrm{CD} 14^{-} \mathrm{CD} 16^{-} \mathrm{CD} 11 \mathrm{c}^{+} \mathrm{HLA}-\mathrm{DR}{ }^{+}\right)$, respectively (Supplementary Fig. 13b-e). We compared $10 \mathrm{nM}$ bortezomib to $1 \mu \mathrm{M}$ PKS21221 as these concentrations gave comparable reductions in ASCs in the differentiation culture (Supplementary Fig. 12B). Bortezomib was more cytotoxic at $10 \mathrm{nM}$ than PKS2122 at $1 \mu \mathrm{M}$ to resting $\mathrm{CD}^{+}{ }^{+}$cells and $\mathrm{CD} 19^{+}$ B cells in (Supplementary Fig. 13b, c), suggesting that PKS21221 was more selectively cytotoxic than bortezomib for activated $B$ cells and ASCs.
PKS21221 inhibits proliferation of $T$ cells. As we observed selective cytotoxicity of PKS21221 in activated B cells and ASCs compared to non-activated B cells, we asked whether this is the case in proliferating $\mathrm{T}$ cells. We activated human $\mathrm{T}$ cells to proliferate with anti-CD3 and anti-CD28 in the presence or absence of PKS21221 or bortezomib. Both compounds inhibited proliferation of $\mathrm{T}$ cells in a dose-dependent manner (Fig. 6a, b). To examine whether these inhibitors are cytotoxic preferentially to proliferating cells, we triggered T-cell proliferation with antiCD3/anti-CD28 for 4 days (in the absence of inhibitors), treated the cells with PKS21221 for the next $24 \mathrm{~h}$ and determined cell survival with Annexin V and 7-AAD (Fig. 6c, d). Both PK21221 and bortezomib induced apoptosis and cell death to a greater extent in proliferating $\mathrm{T}$ cells than in non-proliferating $\mathrm{T}$ cells, suggesting that proteasome inhibitors preferentially affect survival of activated, proliferating $\mathrm{T}$ cells.

\section{Discussion}

These results introduce a versatile new scaffold of proteasome inhibitors, describe their potential for optimization for selective inhibition of the immunoproteasome over the constitutive proteasome, present the cryo-EM structure of the human immunoproteasome in complex with an inhibitor, and demonstrate the inhibitors' ability to kill myeloma cells, activated human B cells and ASCs in vitro, and to a lesser extent, proliferating human $\mathrm{T}$ cells.

To our knowledge, the structure of the human immunoproteasome has not been previously reported, nor has a structure previously been reported of any immunoproteasome with a kinetically noncompetitive inhibitor. The enhanced interior resolution allowed us to confidently interpret the favorable binding of PKS21004 in $\beta 5 \mathrm{i}$ over $\beta 5 \mathrm{c}$ and is guiding structureactivity relationship studies to improve potency and selectivity. How active site-binding AsnEDAs kinetically behave like noncompetitive inhibitors remains to be determined. Active sitedirected noncompetitive inhibitors of other enzymes have been reported and mechanistic investigations have suggested several possibilities $^{32}$. A molecular dynamics study of the proteasome suggested that conformational changes are induced upon binding of an inhibitor to one $\beta 5$ that are propagated to the trans- $\beta 5$ in the other $\beta$ ring through $\beta 4^{38}$. That mechanism does not appear to apply here, as the Hill coefficient constant is $\sim 1$ for the inhibition of either $\beta 5 \mathrm{i}$ or $\beta 5 \mathrm{c}$ by either PKS21004 or PKS21221, 
Table 3 Inhibition of intracellular proteasomal activities and cytotoxicity of PKS21221

\section{$\mathrm{IC50}^{\mathrm{a}}$ (nM)}

$\mathrm{EC}^{\mathrm{a}} \mathrm{a}(\mathrm{nM})$

\begin{tabular}{llllll}
\hline Karpas $\beta 5 \mathrm{i}$ & Karpas $\beta 5_{(\mathrm{i}+\mathrm{c})}$ & HepG2 $\beta 5 \mathrm{c}$ & MM.1S & RPMI 8226 & Karpas \\
$172 \pm 33$ & $118 \pm 33$ & $1833 \pm 138$ & $78 \pm 30$ & $66 \pm 6$ & $740 \pm 145$ \\
\hline
\end{tabular}

$\beta 5 i$ activity was assayed with (Ac-ANW) $)_{2}-R 110$

$\beta 5_{(i+c)}$ and $\beta 5 c$ activity were assayed with suc-LLVY-luciferin

$\beta 5_{(i+c)}$ defines the total chymotryptic activity inside the Karpas cells

a Data are means + SEM of three to four independent experiments

respectively. It remains to be investigated if binding of inhibitor to one $\beta 5$ only affects the hydrolytic activity of the trans- $\beta 5$, but not its binding of substrate.

A growing body of evidence shows that selective inhibition of the immunoproteasome is beneficial in immunity-related diseases without the mechanism-based toxicity of indiscriminate inhibition of all proteasomes. Some immune cells are highly susceptible to proteasome inhibition. For example, plasmacytoid dendritic cells $^{39}$ and plasma cells ${ }^{40}$ are hypersensitive to bortezomib. However, differential hypersensitivity of immune cells towards selective $\beta 5 \mathrm{i}$ inhibition is unknown. Here we show that malignant myeloma cells are susceptible to $\beta 5$ i inhibition. Furthermore, we found that differentiated ASCs and proliferating T cells are susceptible to $\beta 5 \mathrm{i}$ inhibition. This suggests the potential clinical utility of $\beta 5$ i-selective inhibitors in the treatment of diseases with dysregulated B-cell activity, such as antibody-mediated allograft rejection or autoantibody-exacerbated disorders such as lupus and type 1 diabetes. Such effects may be enhanced by inhibition of the proliferation of helper T cells.

To our knowledge, this is a reported example of active sitedirected noncompetitive proteasome inhibitors. Unlike competitive inhibitors whose intracellular activity is expected to diminish if substrates accumulate, noncompetitive inhibitors are expected to retain a similar degree of inhibitory activity with substrate accumulation, or even increased activity, given that the binding of AsnEDA compounds to $\beta 5 c$ and $\beta 5 \mathrm{i}$ was enhanced by substrate.

\begin{abstract}
Methods
Reagents. The human $20 \mathrm{~S}$ immunoproteasome core sample was purchased from Boston Biochem. The human i-20s at a concentration of $1.5 \mathrm{mg}$ per $\mathrm{ml}$ was incubated with PKS21004 dissolved in DMF with a molar ratio of 1:250 ratio for $1 \mathrm{~h}$ at $37^{\circ} \mathrm{C}$, then the mixture was diluted in $50 \mathrm{mM}$ HEPES, pH 7.6, $100 \mathrm{mM} \mathrm{NaCl}$ and $1 \mathrm{mM}$ dithiotreitol to a final concentration of $0.1 \mathrm{mg}$ per $\mathrm{mL}$ for cryo-EM study. Antibodies used for flow cytometry are described as below (clone, company): CD3 (UCHT1, Biolegend), CD4 (OKT4, Biolegend), CD8 (SK1, Biolegend), CD11c (3.9, Biolegend), CD14 (RMO52, Beckman Coulter), CD16 (3G8, Biolegend), CD19 (HIB19, BD), CD38 (LS198-4-3, Beckman Coulter), CD27 (O323, eBioscience), IgD (IA6-2, eBioscience), HLA-DR (L243, Biolegend), Ki67 (Ki-67, Biolegend).
\end{abstract}

Cryo-EM. 2.8 $\mu \mathrm{L}$ of sample was applied on a carbon-coated Quantifoil R2/1 grid that was freshly glow-discharged in the presence of amylamine to prevent preferred orientations $\mathrm{s}^{41}$. Furthermore, the grid was plunged into liquid ethane using a FEI Vitroblot IV at $5{ }^{\circ} \mathrm{C}$ and $95 \%$ humidity with a blot force setting of 5 , blot time of $3 \mathrm{~s}$ and a wait time of $20 \mathrm{~s}$. The grids were imaged with a $300 \mathrm{kV}$ JEOL JEM-3200FS microscope at a magnification of $\times 30,000$, calibrated pixel size of $1.2 \AA$ and an underfocus range of $1-3 \mu \mathrm{m}$. The images were recorded with a Gatan K2 Summit direct electron detector, where each image is composed of 50 individual frames with an exposure time of $10 \mathrm{~s}$ and a total dose of $60 \mathrm{e}^{-} \AA^{-2}$. A total of 2622 micrographs were collected in three sessions.

Image processing and 3D reconstruction. Images were motion corrected using MotionCor $2^{42}$, followed by removal of bad micrographs by visual screening for drift and high/low defocus. In addition, micrographs where Thon rings did not extend beyond $10 \AA$ were also excluded. Furthermore, particles were picked using swarm from EMAN $2^{43}$ followed by manual removal of false positives and inclusion of false negatives. CTF calculations were done with $\mathrm{GCTF}^{44}$ and particle coordinates were imported to RELION $2.0^{45}$, where all next steps were performed. An initial group of 505,880 raw particles were extracted using a $256 \times 256$ pixel box size and 2D classification was performed. Particles belonging to the best classes were selected and regrouped, yielding a total of 478,709 particles. A preliminary 3D classification and refinement was performed using the human constitutive $20 \mathrm{~S}$ proteasome $e^{41}$ as initial model and the resulting map was then used for further processing. Further 3D classification followed using a $20 \AA$ low-pass filtered electron density map where initially $\mathrm{C} 1$ symmetry was used to remove bad classes, yielding 378,196 particles, and C2 symmetry for further rounds. Next, 3D refinement was performed and a $4.65 \AA$ map was generated as well as a solvent mask. The process was repeated using a solvent mask for $3 \mathrm{D}$ classification and $3 \mathrm{D}$ refinement, yielding a $4 \AA$ map from 75,017 particles. In addition, movie refinement and particle polishing were performed using a running average of 15 frames. A final round of $3 \mathrm{D}$ refinement using the polished particles and subsequent post processing with correction of the modular transfer function of the detector and negative B factor application resulted in a final map of $3.8 \AA$ where the Fourier shell correlation is 0.143 . Local resolution was estimated with RELION $2.0^{45}$.

Structural modeling with refinement and validation. As an initial model, the crystal structure of human constitutive proteasome (PDB code 5LF1) ${ }^{25}$ was first docked and fitted into the density map using COOT ${ }^{46}$ and Chimera ${ }^{47}$. The structures of $\beta 1, \beta 2$ and $\beta 5$ were then replaced by models of human immunoproteasome subunit $\beta 1 \mathrm{i}, \beta 2 \mathrm{i}$, and $\beta 5 \mathrm{i}$ respectively, which were generated from the crystal structure of the mouse immunoproteasome (PDB code $3 \mathrm{UNH})^{31}$ using the SWISS-MODEL server ${ }^{48}$. The entire human immunoproteasome atomic model was subsequently adjusted manually and rebuilt in COOT. Rigid body and real space refinements of the resulted atomic model were then performed by Phenix ${ }^{49}$ We also performed the reciprocal space refinement procedure with the application of secondary structure and stereochemical constraints in the program Phenix. Finally, the atomic model was validated using MolProbity ${ }^{50}$. Structural figures were prepared in Chimera ${ }^{47}$ and Pymol (https://www.pymol.org). The final model was cross validated using a method described previously ${ }^{51}$, which indicated that our model was not over-fitted.

Cell-based proteasome $\boldsymbol{\beta 5}$ activity assay. Intracellular proteasomal activity and inhibition assay were performed as reported ${ }^{14}$. Karpas $1106 \mathrm{P}(80,000$ cells per well $)$ were plated and incubated with compound at indicated concentrations for $1 \mathrm{~h}$ at $37^{\circ} \mathrm{C}$. The activity of the overall $\beta 5$ activity including $\beta 5 \mathrm{i}$ and $\beta 5 \mathrm{c}$ in each was measured in situ after compound removal with Proteasome-Glo assay kit according to manufacturer's instructions. Luminescence was recorded on a SpectraMax M5 plate reader. Relative percentage of RLU was used to calculate the IC50s.

Malignant B-cell cytotoxicity assays. We used Karpas 1106P B lymphoma cell line (Cat. No. 06072607, Aldrich) $)^{15,16,52}$, HepG2 (HB-8065, ATCC), MM.1S (Cat No. CRL-2974, ATCC). Cells were cultured at $37^{\circ} \mathrm{C}$ in a humidified air $/ 5 \% \mathrm{CO}_{2}$ atmosphere in medium supplemented with $10 \%$ fetal bovine serum, except for the medium for Karpas $1106 \mathrm{P}$ cells which contained $20 \%$ fetal bovine serum, and 100 units per ml penicillin, $100 \mu \mathrm{g}$ per ml streptomycin in RPMI 1640 medium. Karpas $1106 \mathrm{P}$ was used at 80,000 cells per well, MM.1S at 100,000 cells per well, and HepG2 at 12,000 cells per well. Cells plated in a 96-well plate were treated with compounds at indicated concentrations for $72 \mathrm{~h}$ at $37^{\circ} \mathrm{C}$ in a tissue culture incubator with $5 \% \mathrm{CO}_{2}$. Viable cells were counted using Cell-titer Glo assay kit. EC50s were calculated using PRISM (Graphpad).

Antibody-secreting cell differentiation from human PBMC. Human peripheral blood samples were obtained from five different healthy volunteers (BWH Specimen Bank). Peripheral blood mononuclear cells (PBMCs) were isolated by density gradient centrifugation using Lymphoprep (StemCell Technologies). PBMCs were cultured at $1.5 \times 10^{6}$ cells per mL in RPMI 1640 media (Lonza), supplemented with $10 \%$ human serum (GemCell) (10\% HS-RPMI), recombinant human IL-2 $10 \mathrm{ng}$ per mL (or $100 \mathrm{U}$ per ml, BioLegend) and R848 $1 \mu \mathrm{g}$ per mL (Cayman Chemicals) for 5 days, at $37^{\circ} \mathrm{C}, 5 \% \mathrm{CO}_{2}$. PKS21221 in DMSO, or DMSO only for control, was added to the culture media on day 5 , and cells were incubated for additional 12, 24, and $48 \mathrm{~h}$. Cells were then stained with surface marker antibodies and analyzed by flow cytometry. The viability and apoptosis were assessed using Annexin V Apoptosis Detection Kit (BD Biosciences).

Viability assay of human PBMC. Human PBMCs were obtained from 5 different healthy volunteers as above. Isolated PBMCs were incubated in 10\% HS-RPMI at $5 \times 10^{5}$ cells per $200 \mu \mathrm{l}$ per well in 96 -well round bottom plate (Corning), for 12,24 , 
a Gated on viable, CD19+ cells

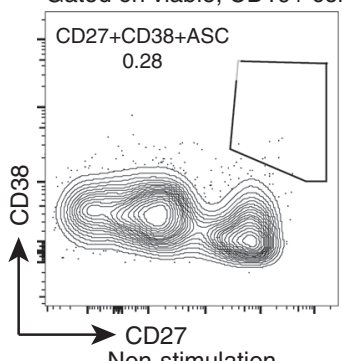

Non-stimulation

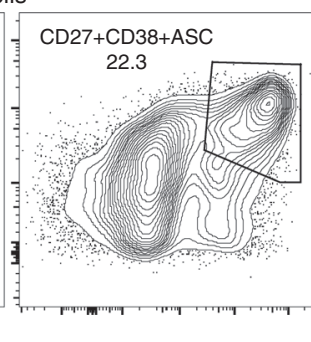

IL-2+R848

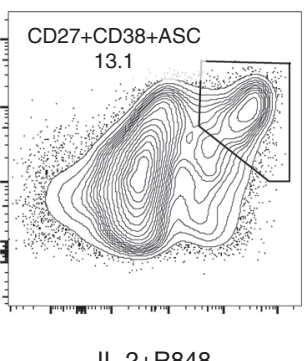

IL-2+R848

+PKS212210.33 $\mu \mathrm{M}$

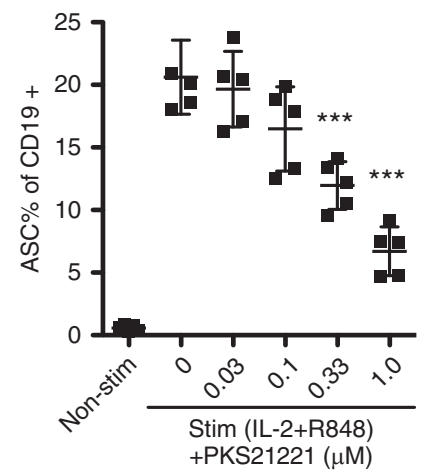

C

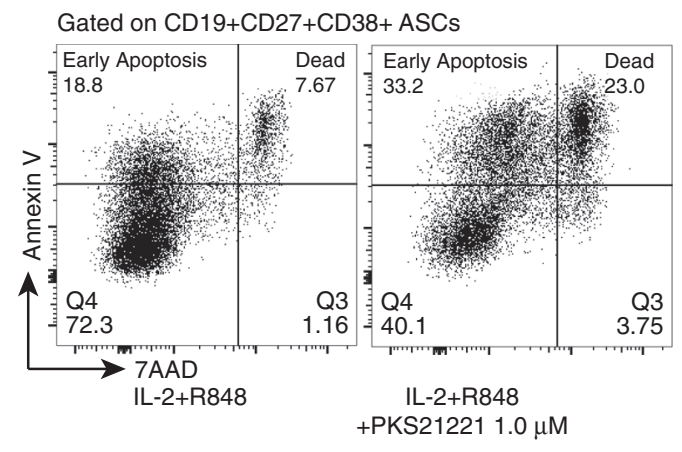

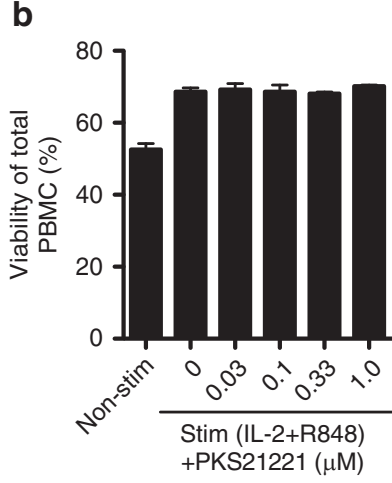
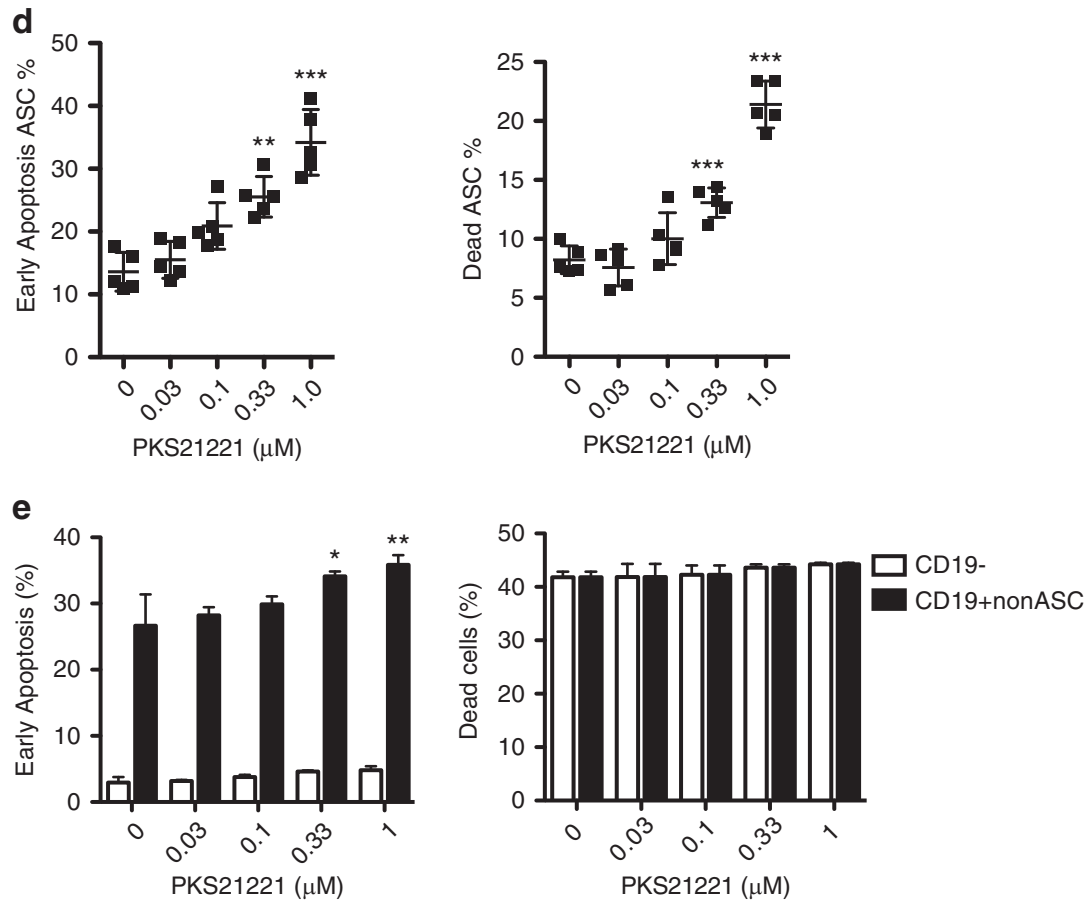

Fig. 5 PKS21221 induced apoptosis and cell death in differentiated antibody-secreting cells (ASCs) and CD19 ${ }^{+}$B cells. PBMCs were cultured with or without IL-2/R848 for 5 days, followed by 12-h incubation with PKS21221 at different concentrations. a Representative flow cytometry plots of cells treated with IL2 and R848 in the presence and absence of PKS21221. IL-2 and R848 differentiated B cells into CD27 ${ }^{+}$CD38 ${ }^{+}$antibody-secreting cells (ASCs). PKS21221 reduced the percentage of ASCs. b Viability of total PBMCs using 7-aminoactinomycin D (7-AAD). 12-h treatment with PKS21221 did not affect overall viability of PBMCs. c $A$ representative plot of apoptosis and viability assay using $A n n e x i n ~ V$ and $7-A A D$. Annexin $V^{+} 7-A A D^{-}$cells were referred as "Early apoptosis" population, and Annexin $\mathrm{V}^{+} 7-\mathrm{AAD}^{+}$cells were referred as "Dead" population. $\mathbf{d}$ Percentage of early apoptotic ASCs (left) and dead ASCs (right), after 12-h incubation with PKS21221. PKS21221 treatment induced apoptotic cell death in a dose-dependent manner. e Early apoptotic (left) and dead (right) populations in CD19- non-B cells and CD19 ${ }^{+}$non-ASCs. Experiments were repeated on PBMCs from 5 donors in 5 separate experiments, each data points in $\mathbf{b}$ and $\mathbf{e}$, were the mean + SEM of three technical replicates. ${ }^{\star} p<0.05$, ${ }^{\star \star} p<0.01$, ${ }^{\star \star \star} p<0.001$, by $t$-test compared with non-treated group 
a

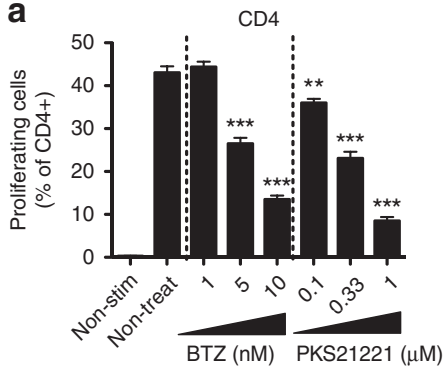

b

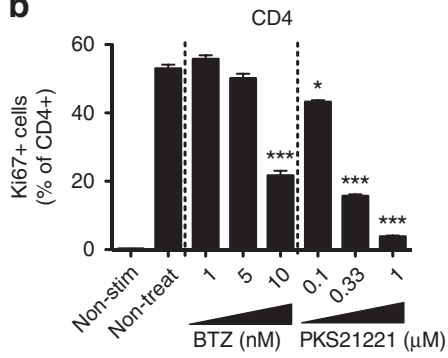

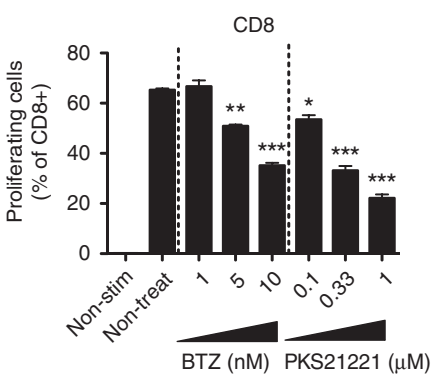

C
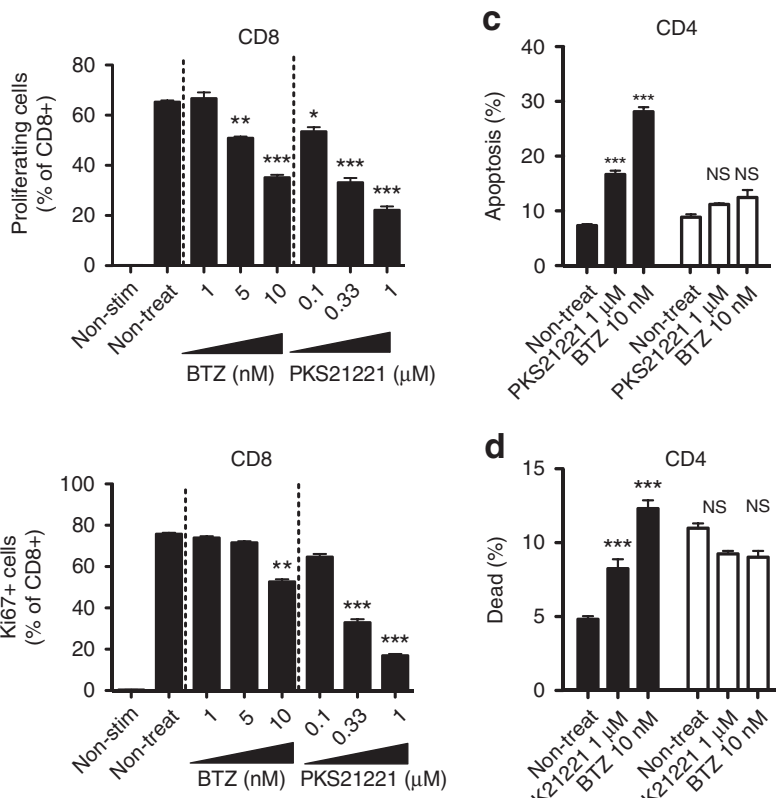

d

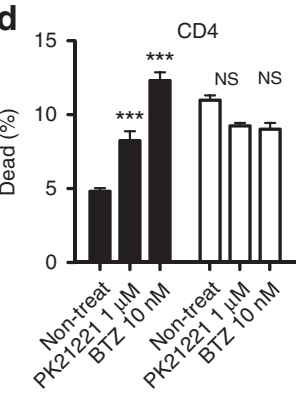

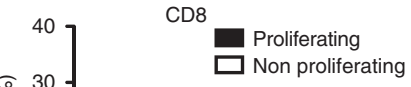
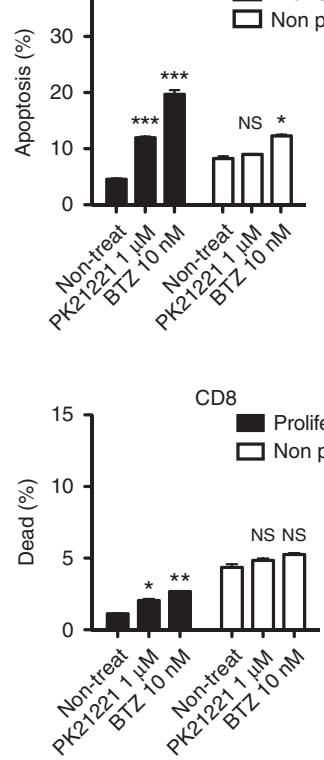

Fig. 6 Effect of PKS21221 and bortezomib (BTZ) on T-cell proliferation. T cells were isolated from human PBMC and stimulated with anti-CD3 (1 $\mu$ g per ml) and anti-CD28 ( $1 \mu \mathrm{g}$ per $\mathrm{ml})$ for 4 days. The gating strategy for flow cytometry is shown in Supplementary Fig. 14. a The percentage of proliferating cell \% of CD4 (left) and CD8 (right) T cells in the presence of different concentrations of BTZ or PKS21221 is shown (mean \pm SEM of triplicates). $\mathbf{b}$ The percentage of $\mathrm{Ki} 67^{+}$cell \% of CD4 (left) and CD8 (right) T cells in the presence of different concentrations of BTZ or PKS21221 is shown (mean \pm SEM of triplicates). Both agents inhibited the proliferation of $T$ cells in a dose-dependent manner. $\mathbf{c}$ and $\mathbf{d}$ : $T$ cells were stimulated with anti-CD3 and anti-CD28 for 4 days, followed by incubation with PKS21221 (1 $\mu \mathrm{M})$ or BTZ $(10 \mathrm{nM})$ for $24 \mathrm{~h}$. Apoptosis $\mathbf{c}$ and viability $\mathbf{d}$ were assessed in proliferating and nonproliferating population of T cells. PKS21221 and BTZ induced apoptosis more significantly in proliferating cells, both in CD4 + and CD8 + T cells. Data are representative results with cells from three different healthy donors, each tested in two different independent experiments. ${ }^{\star} p<0.05,{ }^{\star \star} p<0.01$, ${ }^{\star \star \star} p<0.001$, by $t$-test compared with non-treated group

$48 \mathrm{~h}$, in the presence of different concentrations of PKS21221 or Bortezomib (Selleckchem). The viability of different populations of PBMCs, such as T cells $\left(\mathrm{CD}^{+}\right)$, B cells $\left(\mathrm{CD} 19^{+} \mathrm{CD}^{-} \mathrm{CD} 14^{-}\right)$, monocytes $\left(\mathrm{CD} 14^{+}\right)$, dendritic cells $(\mathrm{CD} 3$ $\left.{ }^{-} \mathrm{CD} 19^{-} \mathrm{CD} 14^{-} \mathrm{CD} 16^{-} \mathrm{HLADR}^{+} \mathrm{CD} 11 \mathrm{c}^{+}\right)$, were assessed by flow cytometry using Fixable Viability dye (Themo Fisher).

In vitro $\mathbf{T}$ cells activation and proliferation. $\mathrm{T}$ cells were isolated from human PBMCs using Human T-cell Isolation kit (StemCell Technologies, Cat\#17951). The purity of $\mathrm{T}$ cells was greater than $90 \%$ as confirmed by flow cytometry. Cells were counted and loaded with $5 \mu \mathrm{M}$ CellTrace Violet (Thermo Fisher) in phosphate buffered saline (PBS) for $10 \mathrm{~min}$ at $37^{\circ} \mathrm{C}$, followed by wash in $10 \%$ fetal calf serum (FCS)-supplemented RPMI 1640 media. T cells were cultured in 10\% HS-RPMI, with anti-CD3 $(1 \mu \mathrm{g}$ per $\mathrm{mL}$, eBioscience) and anti-CD28 $(1 \mu \mathrm{g}$ per $\mathrm{mL}$, eBioscience), in the presence or absence of different concentration of PKS21221 or Bortezomib (Selleckchem). In some experiments, T cells were cultured in 10\% HSRPMI / anti-CD3 / anti-CD28 for 4 days and then treated with PKS21221 or Bortezomib (Selleckchem) for 12, 24, $48 \mathrm{~h}$. The viability and apoptosis were assessed using Annexin V Apoptosis Detection Kit (BD Biosciences). The experiments were performed in cells from three different healthy volunteer donors.

Flow cytometry. Cells were stained with surface marker antibodies at pre-titrated concentrations, in $2 \%$ FCS-containing PBS on ice for $30 \mathrm{~min}$. For intracellular staining of Ki67, surface-stained cells were washed with $2 \%$ FCS-PBS, followed by incubation in fixation/permeabilization buffer (eBioscience) on ice for $45 \mathrm{~min}$ and intracellular staining in permeabilization buffer (eBioscience) for $30 \mathrm{~min}$ at room temperature. Cells were analyzed using FACS CantoII (BD Biosciences) and BD FACS Diva software (BD Bioscience). Data were analyzed by FlowJo software (version 10, Tree Star). Gating strategies for each experiment were provided in the supplementary information.

Data availability. All the relevant data are available within the paper and its Supplementary Information file or from the corresponding authors upon reasonable request. The cryo-EM 3D map has been deposited in the EMDB database with accession code EMD-7010. The corresponding atomic model has been deposited in the RCSB PDB with accession code 6AVO.
Received: 27 May 2017 Accepted: 13 October 2017

Published online: 22 November 2017

\section{References}

1. Goldberg, A. L. Functions of the proteasome: from protein degradation and immune surveillance to cancer therapy. Biochem. Soc. Trans. 35, 12-17 (2007),

2. Baumeister, W., Walz, J., Zuhl, F. \& Seemuller, E. The proteasome: paradigm of a self-compartmentalizing protease. Cell 92, 367-380 (1998).

3. Borissenko, L. \& Groll, M. 20 S proteasome and its inhibitors: crystallographic knowledge for drug development. Chem. Rev. 107, 687-717 (2007).

4. Huber, E. M. \& Groll, M. Inhibitors for the immuno- and constitutive proteasome: current and future trends in drug development. Angew. Chem. Int. Ed. Engl. 51, 8708-8720 (2012).

5. Lansdell, T. A. et al. Noncompetitive modulation of the proteasome by imidazoline scaffolds overcomes bortezomib resistance and delays MM tumor growth in vivo. ACS Chem. Biol. 8, 578-587 (2013).

6. Azevedo, L. M. et al. Inhibition of the human proteasome by imidazoline scaffolds. J. Med. Chem. 56, 5974-5978 (2013).

7. Lawrence, H. R. et al. Synthesis and biological evaluation of naphthoquinone analogs as a novel class of proteasome inhibitors. Bioorg. Med. Chem. 18, 5576-5592 (2010).

8. Lin, G. et al. N,C-Capped dipeptides with selectivity for mycobacterial proteasome over human proteasomes: role of $\mathrm{S} 3$ and $\mathrm{S} 1$ binding pockets. J. Am. Chem. Soc. 135, 9968-9971 (2013).

9. Lin, G. et al. Mycobacterium tuberculosis prcBA genes encode a gated proteasome with broad oligopeptide specificity. Mol. Microbiol. 59, 1405-1416 (2006).

10. Lin, G., Li, D., Chidawanyika, T., Nathan, C. \& Li, H. Fellutamide B is a potent inhibitor of the Mycobacterium tuberculosis proteasome. Arch. Biochem. Biophys. 501, 214-220 (2010).

11. Lin, G. et al. Inhibitors selective for mycobacterial versus human proteasomes. Nature 461, 621-626 (2009).

12. Lin, G., Tsu, C., Dick, L., Zhou, X. K. \& Nathan, C. Distinct specificities of Mycobacterium tuberculosis and mammalian proteasomes for $\mathrm{N}$-acetyl tripeptide substrates. J. Biol. Chem. 283, 34423-34431 (2008). 
13. Fan, H., Angelo, N. G., Warren, J. D., Nathan, C. F. \& Lin, G. Oxathiazolones selectively inhibit the human immunoproteasome over the constitutive proteasome. ACS Med. Chem. Lett. 5, 405-410 (2014).

14. Sula Karreci, E. et al. Brief treatment with a highly selective immunoproteasome inhibitor promotes long-term cardiac allograft acceptance in mice. Proc. Natl Acad. Sci. USA, doi:10.1073/pnas.1618548114 (2016).

15. Singh, P. K. et al. Immunoproteasome $\beta 5$ i-selective dipeptidomimetic inhibitors. ChemMedChem, doi:10.1002/cmdc.201600384 (2016).

16. Wilhelm, M. et al. Mass-spectrometry-based draft of the human proteome. Nature 509, 582-587 (2014).

17. Kim, M. S. et al. A draft map of the human proteome. Nature 509, 575-581 (2014).

18. Tanaka, K. Role of proteasomes modified by interferon-gamma in antigen processing. J. Leukoc. Biol. 56, 571-575 (1994).

19. Palombella, V. J. et al. Role of the proteasome and NF-kappaB in streptococcal cell wall-induced polyarthritis. Proc. Natl Acad. Sci. USA 95, 15671-15676 (1998).

20. Kalim, K. W., Basler, M., Kirk, C. J. \& Groettrup, M. Immunoproteasome subunit LMP7 deficiency and inhibition suppresses Th1 and Th17 but enhances regulatory T cell differentiation. J. Immunol. 189, 4182-4193 (2012).

21. Raghavan, R. et al. Bortezomib in kidney transplantation. J. Transplant. 2010 doi:10.1155/2010/698594 (2010).

22. Alexander, T. et al. The proteasome inhibitior bortezomib depletes plasma cells and ameliorates clinical manifestations of refractory systemic lupus erythematosus. Ann. Rheum Dis. 74, 1474-1478 (2015).

23. Kincaid, E. Z. et al. Mice completely lacking immunoproteasomes show major changes in antigen presentation. Nat. Immunol. 13, 129-135 (2012)

24. Muchamuel, T. et al. A selective inhibitor of the immunoproteasome subunit LMP7 blocks cytokine production and attenuates progression of experimental arthritis. Nat. Med. 15, 781-787 (2009).

25. Schrader, J. et al. The inhibition mechanism of human $20 \mathrm{~S}$ proteasomes enables next-generation inhibitor design. Science 353, 594-598 (2016).

26. Cui, H. et al. Structural elucidation of a non-peptidic inhibitor specific for the human immunoproteasome. Chembiochem, doi:10.1002/cbic.201700021 (2017).

27. Dubiella, C., Baur, R., Cui, H., Huber, E. M. \& Groll, M. Selective inhibition of the immunoproteasome by structure-based targeting of a non-catalytic cysteine. Angew. Chem. Int. Ed. Engl 54, 15888-15891 (2015).

28. Blackburn, C. et al. Characterization of a new series of non-covalent proteasome inhibitors with exquisite potency and selectivity for the 20S beta5subunit. Biochem. J. 430, 461-476 (2010).

29. Copeland, R. A. in: Evaluation of Enzyme Inhibitors in Drug Discovery: A Guide for Medicinal Chemists and Pharmacologists, 2nd Edition, 1-538, doi:10.1002/9781118540398 (2013).

30. Ozcan, S. et al. Oxadiazole-isopropylamides as potent and noncovalent proteasome inhibitors. J. Med. Chem. 56, 3783-3805 (2013).

31. Huber, E. M. et al. Immuno- and constitutive proteasome crystal structures reveal differences in substrate and inhibitor specificity. Cell 148, 727-738 (2012).

32. Blat, Y. Non-competitive inhibition by active site binders. Chem. Biol. Drug. Des. 75, 535-540 (2010)

33. Parlati, F. et al. Carfilzomib can induce tumor cell death through selective inhibition of the chymotrypsin-like activity of the proteasome. Blood 114, 3439-3447 (2009).

34. Richardson, P. G. et al. Bortezomib or high-dose dexamethasone for relapsed multiple myeloma. N. Engl. J. Med. 352, 2487-2498 (2005).

35. Ichikawa, H. T. et al. Beneficial effect of novel proteasome inhibitors in murine lupus via dual inhibition of type I interferon and autoantibody-secreting cells. Arthritis Rheum. 64, 493-503 (2012).

36. Jahnmatz, M. et al. Optimization of a human IgG B-cell ELISpot assay for the analysis of vaccine-induced B-cell responses. J. Immunol. Methods 391, 50-59 (2013).

37. Crotty, S., Aubert, R. D., Glidewell, J. \& Ahmed, R. Tracking human antigenspecific memory B cells: a sensitive and generalized ELISPOT system. J. Immunol. Methods 286, 111-122 (2004).

38. Arciniega, M., Beck, P., Lange, O. F., Groll, M. \& Huber, R. Differential global structural changes in the core particle of yeast and mouse proteasome induced by ligand binding. Proc. Natl Acad. Sci. USA 111, 9479-9484 (2014).

39. Hirai, M. et al. Bortezomib suppresses function and survival of plasmacytoid dendritic cells by targeting intracellular trafficking of Toll-like receptors and endoplasmic reticulum homeostasis. Blood 117, 500-509 (2011).

40. Neubert, K. et al. The proteasome inhibitor bortezomib depletes plasma cells and protects mice with lupus-like disease from nephritis. Nat. Med. 14, 748-755 (2008)

41. da Fonseca, P. C. \& Morris, E. P. Cryo-EM reveals the conformation of a substrate analogue in the human $20 \mathrm{~S}$ proteasome core. Nat. Commun. 6, 7573 (2015).

42. Zheng, S. Q. et al. MotionCor2: anisotropic correction of beam-induced motion for improved cryo-electron microscopy. Nat. Methods 14, 331-332 (2017).
43. Tang, G. et al. EMAN2: an extensible image processing suite for electron microscopy. J. Struct. Biol. 157, 38-46 (2007).

44. Zhang, K., Gctf \& Real-time, C. T. F. determination and correction. J. Struct. Biol. 193, 1-12 (2016).

45. Kimanius, D., Forsberg, B. O., Scheres, S. H. \& Lindahl, E. Accelerated cryo-EM structure determination with parallelisation using GPUs in RELION-2. Elife 5, doi:10.7554/eLife.18722 (2016)

46. Emsley, P. \& Cowtan, K. Coot: model-building tools for molecular graphics. Acta Crystallogr. D Biol. Crystallogr. 60, 2126-2132 (2004).

47. Pettersen, E. F. et al. UCSF Chimera-a visualization system for exploratory research and analysis. J. Comput. Chem. 25, 1605-1612 (2004).

48. Biasini, M. et al. SWISS-MODEL: modelling protein tertiary and quaternary structure using evolutionary information. Nucleic Acids Res. 42, W252-W258 (2014).

49. Adams, P. D. et al. PHENIX: a comprehensive Python-based system for macromolecular structure solution. Acta Crystallogr. D Biol. Crystallogr. 66, 213-221 (2010).

50. Chen, V. B. et al. MolProbity: all-atom structure validation for macromolecular crystallography. Acta Crystallogr. D Biol. Crystallogr. 66, 12-21 (2010).

51. Amunts, A. et al. Structure of the yeast mitochondrial large ribosomal subunit Science 343, 1485-1489 (2014).

52. Blackburn, C. et al. Optimization of a series of dipeptides with a P3 threonine residue as non-covalent inhibitors of the chymotrypsin-like activity of the human 20 S proteasome. Bioorg. Med. Chem. Lett. 20, 6581-6586 (2010).

\section{Acknowledgements}

This work was supported by Alliance for Lupus Research (Award 255848, G.L.), the Daedalus Fund for Innovation (2014 G.L.) of Weill Cornell Medicine, the Milstein Program in Translational Medicine and Chemical Biology (C.N.), American Heart Association (AHA Award 13FTF17000018 to J.A.), American Diabetes Association (ADA award 1-17-IBS-206 to J.A.) and the National Institutes of Health (R01 AI70285 and U24 GM116787 to H.L.; R21 AI101393 and AI123794 to G.L.; T32 DK007527 to N M.). We thank Dr J. David Warren at The Abby and Howard P. Milstein Synthetic Chemistry Core Facility at Weill Cornell Medicine for assistance. We thank Ms. Rong Wang and Dr George Sukenick at Memorial Sloan Kettering Cancer Center for assistance with high-resolution mass spectrometry. We thank Dr Jingchuan Sun, currently at University of Pennsylvania, for help with cryo-EM grid preparation and initial grid screening. We thank Dr Wah Chiu at National Center for Macromolecular Imaging and Marrs McLean Department of Biochemistry and Molecular Biology, Baylor College of Medicine for support in cyro-EM data collection. The Department of Microbiology and Immunology is supported by the William Randolph Hearst Foundation.

\section{Author contributions}

R.S., L.B., P.K. and N.M. conducted the majority of the experiments and contributed equally to the study. K.Z., assisted cryo-EM studies. H.F., W.Z., Y.Z. and X.J. conducted biochemical characterization of compounds. J.A. assisted N.M in FACS. All authors discussed the data and commented on the manuscript. C.N., H.L., J.A. and G.L. conceived and directed the project.

\section{Additional information}

Supplementary Information accompanies this paper at doi:10.1038/s41467-017-01760-5

Competing interests: The authors declare no competing financial interests.

Reprints and permission information is available online at http://npg.nature.com/ reprintsandpermissions/

Publisher's note: Springer Nature remains neutral with regard to jurisdictional claims in published maps and institutional affiliations.

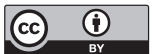

Open Access This article is licensed under a Creative Commons Attribution 4.0 International License, which permits use, sharing, adaptation, distribution and reproduction in any medium or format, as long as you give appropriate credit to the original author(s) and the source, provide a link to the Creative Commons license, and indicate if changes were made. The images or other third party material in this article are included in the article's Creative Commons license, unless indicated otherwise in a credit line to the material. If material is not included in the article's Creative Commons license and your intended use is not permitted by statutory regulation or exceeds the permitted use, you will need to obtain permission directly from the copyright holder. To view a copy of this license, visit http://creativecommons.org/ licenses/by/4.0/.

(C) The Author(s) 2017 